\title{
Contraction Anisocoria: Segregation, Summation, and Saturation in the Pupillary Pathway
}

\author{
Corinne F. Carle, Ted Maddess, and Andrew C. James
}

\begin{abstract}
Purpose. To investigate the neural basis of contraction anisocoria and any implications for assessments using pupillary responses, through analysis of topographic variation in amplitudes of direct and consensual pupil responses.
\end{abstract}

MeTHODs. Direct and consensual pupillary contraction amplitudes were analyzed from six studies in which 120 normal subjects were tested with 24 different stimulus variants. The dichoptically presented multifocal stimulus arrays subtended $\pm 30^{\circ}$ of visual field but varied in color (achromatic or yellow), number of test regions (24 to 60/eye), mean regional presentation interval ( 0.25 to $16 \mathrm{~s})$, and pulse time-course (33 to $150 \mathrm{~ms}$, flickered or steady). The $290 \mathrm{~cd} / \mathrm{m}^{2}$ test-regions were displayed on a $10 \mathrm{~cd} / \mathrm{m}^{2}$ background. Ratios between mean direct and consensual responses were calculated for each region. Results were quantified using multivariate linear analysis.

REsults. Direct responses within the temporal hemifield were significantly larger than consensual for all stimulus protocols. Across the 24 protocols these differences ranged between $13.8 \%\left(t_{1415}=3.06, P<0.01\right)$ and $27.0 \%\left(t_{990}=5.72, P<\right.$ $0.0001)$. Differences in the nasal field were mostly non-significant. Contraction amplitudes varied systematically across the visual field. By contrast, direct/consensual ratios were markedly uniform within each hemifield.

Conclusions. The distribution of signal from the pretectal olivary nuclei to each Edinger-Westphal nucleus differs depending on the hemifield being stimulated. A simple model incorporating segregation and summation of afferent signals, and differing saturation of midbrain pathways is proposed. This appears to explain inconsistent observations in the literature and predicates the need for separate assessment of direct and consensual responses at hemifield or better resolution. (Invest Ophthalmol Vis Sci. 2011;52:2365-2371) DOI:10.1167/iovs.106335

$T^{t}$ is reasonably well recognized that, even in the absence of 1 pathology, presentation of a transient light stimulus to a single eye will produce small differences between direct and consensual pupillary responses. ${ }^{1,2}$ While in general, larger direct than consensual responses have been reported, the nature

From the ARC Centre of Excellence in Vision Science, Research School of Biology, The Australian National University, Canberra, Australia.

Supported by the Australian Research Council through the ARC Centre of Excellence in Vision Science (CE0561903).

Submitted for publication August 4, 2010; accepted October 26 , 2010.

Disclosures: C.F. Carle, Seeing Machines Ltd (F); T. Maddess, Seeing Machines Ltd (F, I, C, P, R); A.C. James, Seeing Machines Ltd (F, I, C, P, R)

Corresponding author: Corinne F. Carle, Research School of Biology, The Australian National University, GPO Box 475, Canberra, ACT 2601, Australia; corinne.carle@anu.edu.au. of this contraction anisocoria differs if stimulation is restricted to a single visual hemifield. ${ }^{3-6}$ Studies have consistently reported observations of larger direct than consensual responses on stimulation of the nasal retina. Stimulation of the temporal retina, however, has produced inconsistent results, with some experiments reporting equivalent responses to temporal stimulation $^{4-6}$ and others that consensual responses are larger than direct. $^{3,7}$ No satisfactory explanation has yet been offered for these conflicting findings.

Characterizing stimulus-response relationships within the neural pathways mediating pupillary contractions has substantial clinical value because pupillary responses are used in the assessment of numerous neurologic and visual pathologies. Published investigations of contraction anisocoria in normal subjects have however, used only hemifield or, at best, quadrant stimulation. The recent development of dichoptic multifocal pupillographic objective perimetry (mfPOP) by this group, ${ }^{8,9}$ with its ability to provide high-resolution mapping of local regional differences between direct and consensual responses, should provide a much richer source of information than has previously been available. Many normal subjects have been tested using mfPOP during its development. We will examine aspects of contraction anisocoria using this very large data set.

\section{Methods}

\section{Subjects}

This research used data collected from 120 normal subjects during their participation in six separate studies centered around the development of mfPOP as a diagnostic tool. For subject and study information see Table 1 . Within each study, all subjects were tested with between two and five different stimulus variants, referred to here as protocols; thus there were six studies encompassing a total of 24 different stimulus protocols.

Subjects in studies 1, 4, 5, and 6 were assessed using commercial instruments (Humphrey [HFA II], Achromatic SITA-Fast perimetry, SWAP Fastpac 24-2 perimetry, Matrix 24-2 perimetry, Stratus OCT; all from Carl Zeiss Meditec Inc., Dublin, CA), slit-lamp biomicroscopy, and applanation tonometry. The remaining smaller studies (studies 2 and 3 ) involved young subjects who had their visual acuity checked and their visual fields assessed using frequency doubling technology (FDT) perimetry. Exclusion criteria for all subjects included the presence of any diagnosed neurologic or ocular pathology as well as previous ocular surgery or refractive errors greater than \pm 6 diopters or $>2$ diopters of cylinder. Subjects were requested not to consume caffeine or alcohol for one hour before testing. Informed written consent was given by all participants after explanation of the nature and possible consequences of the study, according to the ANU Human Experimentation Ethics Committee approval 238/04. This research adhered to the tenets of the Declaration of Helsinki.

\section{Multifocal Pupillographic Objective Perimetry}

Presentation of stimuli and monitoring of pupil diameter was carried out using mfPOP (presented on a prototype of the FDA-cleared True- 
Table 1. Stimulus Characteristics for Each Protocol, and Subject Information for the Six Studies Included in this Analysis

\begin{tabular}{|c|c|c|c|c|c|c|c|c|}
\hline Study & Protocol & $\begin{array}{c}\text { Subjects } \\
(\mathbf{M} / \mathbf{F})\end{array}$ & $\operatorname{Age}^{*}(y)$ & $\begin{array}{c}\text { Test } \\
\text { Regions/Field }\end{array}$ & Color & $\begin{array}{c}\text { Stimulus Duration } \dagger \\
\text { (ms) }\end{array}$ & $\begin{array}{c}\text { Stimulus } \\
\text { Typet }\end{array}$ & $\begin{array}{c}\text { Mean Presentations/ } \\
\text { Second/Region } †\end{array}$ \\
\hline 1 & A-D & $34 / 50$ & $58.1 \pm 9.2$ & 24 & Achromatic & $66^{\mathrm{A}, \mathrm{C}}, 133^{\mathrm{B}, \mathrm{D}}$ & $\mathrm{f} 15^{\mathrm{D}}, \mathrm{f} 30^{\mathrm{A}, \mathrm{B}}, \mathrm{s}^{\mathrm{C}}$ & $4^{\mathrm{A}, \mathrm{C}}, 2^{\mathrm{B}, \mathrm{D}}$ \\
\hline 2 & $A-E$ & $9 / 7$ & $27.2 \pm 9.2$ & 40 & Achromatic & 133 & f $30^{\mathrm{A}-\mathrm{C}}, \mathrm{s}^{\mathrm{D}, \mathrm{E}}$ & $1^{\mathrm{A}}, 1 / 4^{\mathrm{B}, \mathrm{D}}, 1 / 16^{\mathrm{C}, \mathrm{E}}$ \\
\hline 3 & A-E & $9 / 7$ & $27.2 \pm 9.2$ & 60 & Achromatic & 133 & f $30^{\mathrm{A}-\mathrm{C}}, \mathrm{s}^{\mathrm{D}, \mathrm{E}}$ & $1^{\mathrm{A}}, 1 / 4^{\mathrm{B}, \mathrm{D}}, 1 / 16^{\mathrm{C}, \mathrm{E}}$ \\
\hline 4 & A-D & $8 / 11$ & $59.7 \pm 8.7$ & 40 & Achromatic & $33^{\mathrm{B}}, 100^{\mathrm{A}, \mathrm{D}}, 150^{\mathrm{C}}$ & f $20^{\mathrm{A}, \mathrm{C}, \mathrm{D}}, \mathrm{s}^{\mathrm{B}}$ & $1^{\mathrm{A}, \mathrm{B}}, 1 / 4^{\mathrm{D}}, 1 / 16^{\mathrm{C}}$ \\
\hline 5 & $A-B$ & $18 / 24$ & $59.4 \pm 7.7$ & $44 \neq$ & Yellow & 33 & $\mathrm{~s}$ & $1^{\mathrm{A}}, 1 / 4^{\mathrm{B}}$ \\
\hline 6 & A-D & $11 / 10$ & $60.5 \pm 7.0$ & $44 \ddagger$ & Yellow & 33 & $\mathrm{~s}$ & $1^{\mathrm{A}, \mathrm{C}}, 1 / 4^{\mathrm{B}, \mathrm{D}}$ \\
\hline
\end{tabular}

The mfPOP stimulus protocols varied in their number of test regions, with stimulus pulses differing in their color, mean presentation rate, duration, and time-course (stimulus type). A total of 120 subjects' results were included in the analysis, some subjects having participated in more than one study.

* Data shown are mean \pm SD.

† Where variables differ, superscript denotes protocol. F, flicker $(\mathrm{Hz})$; s, steady.

‡ Overlapping region layout.

Field Analyzer; Seeing Machines Ltd., Braddon, CT). This device, under development at the time of these experiments, utilizes concurrent presentation of dichoptic, spatially and temporally sparse, multifocal stimuli at 60 frames/s. ${ }^{10}$ Thus both eyes of each subject were tested concurrently with independent stimuli, permitting direct and consensual responses to be measured at every visual field location. ${ }^{8}$ Infrared light was used to illuminate the subjects' eyes and responses in both pupils were monitored with two video cameras at 30 frames/s/eye, synchronous with stimulus presentation. During testing, subjects fixated a small red cross in the center of the viewing field. Gaze was monitored online and data from blinks and fixation losses was deleted. Stimuli were presented at optical infinity to minimize accommodative responses. Corrective lenses compensated for refractive errors to within 1.5 diopters of the subject's current optical prescription; the stimuli contained no spatial frequencies above 2 cpd (Fig. 1), making them highly tolerant of mis-refraction. ${ }^{11}$
Within each of the six studies, subjects were tested with a randomly ordered series of stimulus protocols, each consisting of eight segments of 30 seconds' duration. Four different stimulus layouts were used in these many experiments; all subtended $60^{\circ}$ of visual angle $\left( \pm 30^{\circ}\right.$ radius from fixation) and comprised between 24 and 60 test regions/eye arranged in a dartboard or overlapping layout (Table 1, Fig. 1). The background illumination was $10 \mathrm{~cd} / \mathrm{m}^{2}$. Individual test regions, when active, displayed a single flickering or steady stimulus pulse at a maximum luminance of $290 \mathrm{~cd} / \mathrm{m}^{2}$. Stimulus duration, color, and temporal spacing of presentations varied between protocols (Table 1).

\section{Data Analysis}

Response waveforms for each test region were extracted from raw pupillary responses using a previously described multiple linear regression method. ${ }^{10,12}$ This provided amplitude estimations of both direct
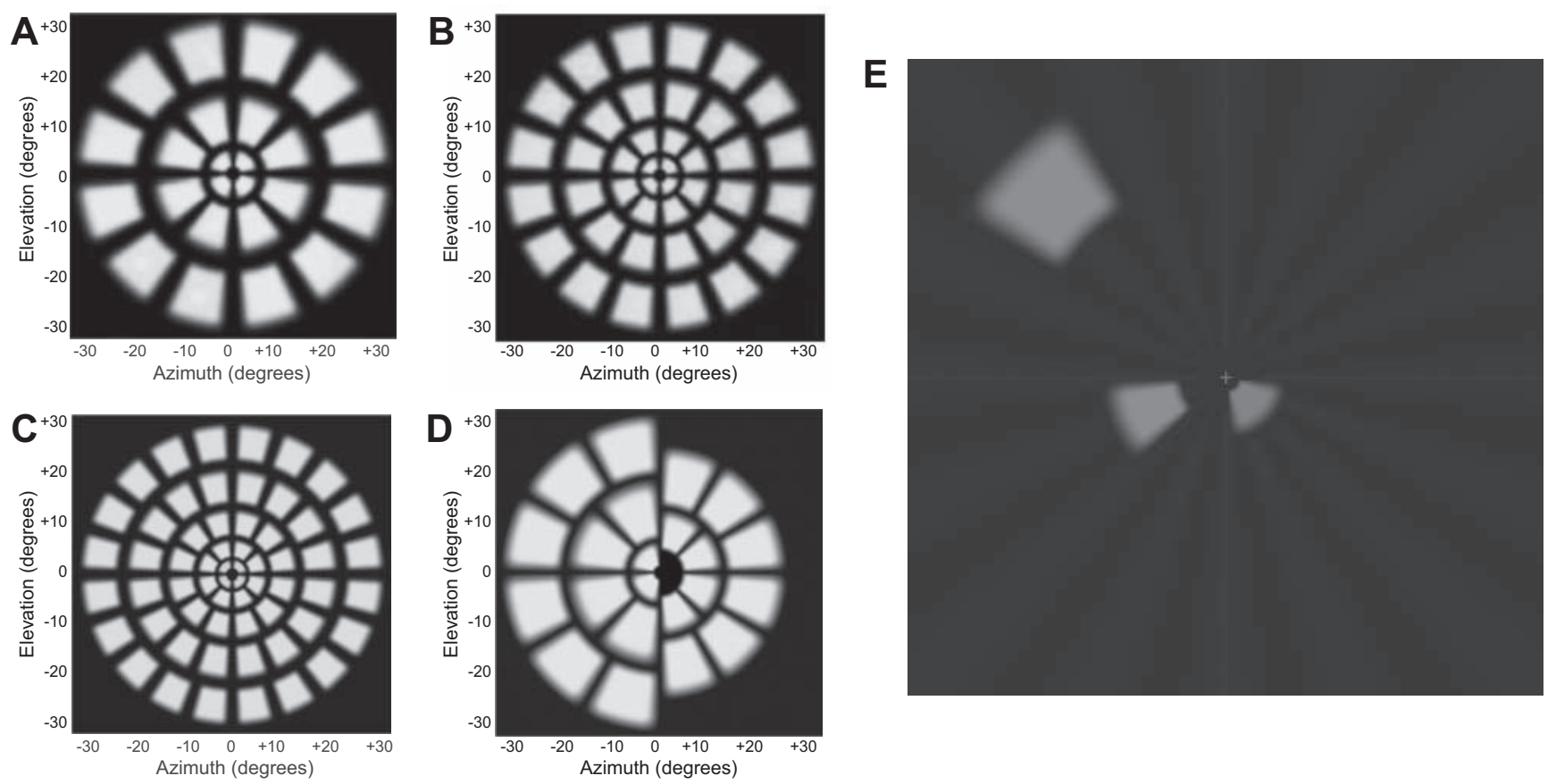

FigURE 1. Stimulus maps showing the layout of test regions for the four different formats used in this series of experiments, and a single frame of a stimulus protocol. (A) A 24-region achromatic stimulus map. (B) A 40-region achromatic stimulus map. (C) A 60-region achromatic stimulus map. (D) A 44-region yellow overlapping stimulus map (shown in greyscale). The layout for this configuration consisted of five rings of slightly overlapping regions (only half of the regions are shown here). (E) A single frame of a stimulus protocol as viewed by a subject. The dichoptic presentation used in these experiments means that some of these test regions are being viewed by one eye, the remainder by the other eye, resulting in this fused percept. The effect of spatially and temporally sparse presentation is also evident in this frame, with only a small subset of regions active at any given time. 
and consensual responses in each test region for each subject and protocol. These estimated responses are effectively a mean impulse response obtained from between 15 and 960 individual stimulus presentations to each region, depending on the presentation rate (Table 1). Due to variation in mean pupil diameter in the general population, comparisons were facilitated by the standardization of contraction amplitudes as described elsewhere. ${ }^{8}$

Data for each right eye visual field was flipped left/right so that all responses were mapped in the same manner as left eyes, with the temporal field on the left side. Variation in direct and consensual responses across visual field locations was investigated by calculating the mean direct and consensual contraction amplitudes for each region and protocol across all eyes, within each of the 24 protocols. Mean direct response amplitudes were then divided by the mean consensual response for the corresponding visual field region to obtain a map of ratios of all visual field regions, for each protocol.

Multivariate linear analysis, incorporating effects for the age and sex of subjects, was used to quantify relationships between direct and consensual responses in temporal and nasal hemifields. The effects of these variables on pupil response amplitudes were multiplicative, so a logarithmic transform was used for this model; this also stabilized the variance. ${ }^{8,9}$ Fitted effects for direct versus consensual responses were then converted to proportional differences, these being reported as the percentage difference from a ratio of 1 . Field intensity was calculated for each protocol by multiplying the stimulus pulse duration (with a compensation for flicker), the stimulus presentation rate per second, the $\log$ of the stimulus luminance, and a factor denoting the density of the stimulus layout relative to the stimulus field. (The abscissa values in Figure 4, being the product of the field intensity and the mean amplitude for each protocol, were scaled in Figure 5 to aid comparison with the proposed model.)

\section{Results}

Visual field maps of pupillary contraction amplitudes displayed relatively smooth and continuous gradients in all protocols, with direct and consensual response amplitudes both being largest in the extreme temporal field and smallest in the inferior nasal field. Examples are shown in Figure 2 (left-hand and central columns). Similar gradients in response amplitude were found for all protocols.

Ratios between regional direct and consensual response amplitudes were consistently larger than 1 in temporal regions, indicating larger direct than consensual responses to stimuli in this hemifield. By contrast, the nasal field locations produced ratios that were uniformly 1 , indicating approximately equivalent direct and consensual response amplitudes (Fig. 2, right). To illustrate this, the mean and SD of the ratios for each hemifield of the two protocols shown in Figure 2 were $1.18 \pm 0.06$ (A) and $1.17 \pm 0.06$ (B) for the temporal field and $1.02 \pm 0.06$ (A) and $0.96 \pm 0.04$ (B) for the nasal field. Thus, the topographic distribution of these ratios did not follow the graduated variation observed with response amplitudes, instead being reasonably homogeneous within each hemifield.

Multivariate linear analysis confirmed these relationships. In the temporal hemifields direct responses were significantly larger $(P<0.01)$ than consensual responses across all 24 protocols (Fig. 3). These temporal visual field effects ranged between $13.8 \%$ larger $\left(t_{1415}=3.06, P<0.01\right)$ and $27.0 \%$ larger $\left(t_{990}=5.72, P<0.0001\right)$ in protocols $4 \mathrm{~B}$ and $2 \mathrm{~B}$, respectively. In the nasal hemifield, differences were less consistent: only three protocols produced ratios differing significantly from 1 , these falling at either extreme of the spread of effect sizes and ranging between direct responses that were $9.6 \%$ smaller than consensual in protocol 3C $\left(t_{1462}=-2.20, P<0.05\right)$ and $6.6 \%$ larger in protocol $6 \mathrm{D}\left(t_{1802}=2.16, P<0.05\right)$. Degrees of freedom for these comparisons ranged from 844 to 15,533 (Fig. 3 , bottom).

\section{Discussion}

Although often described as being mediated by a simple reflex loop, the retinal response that eventually leads to a pupillary
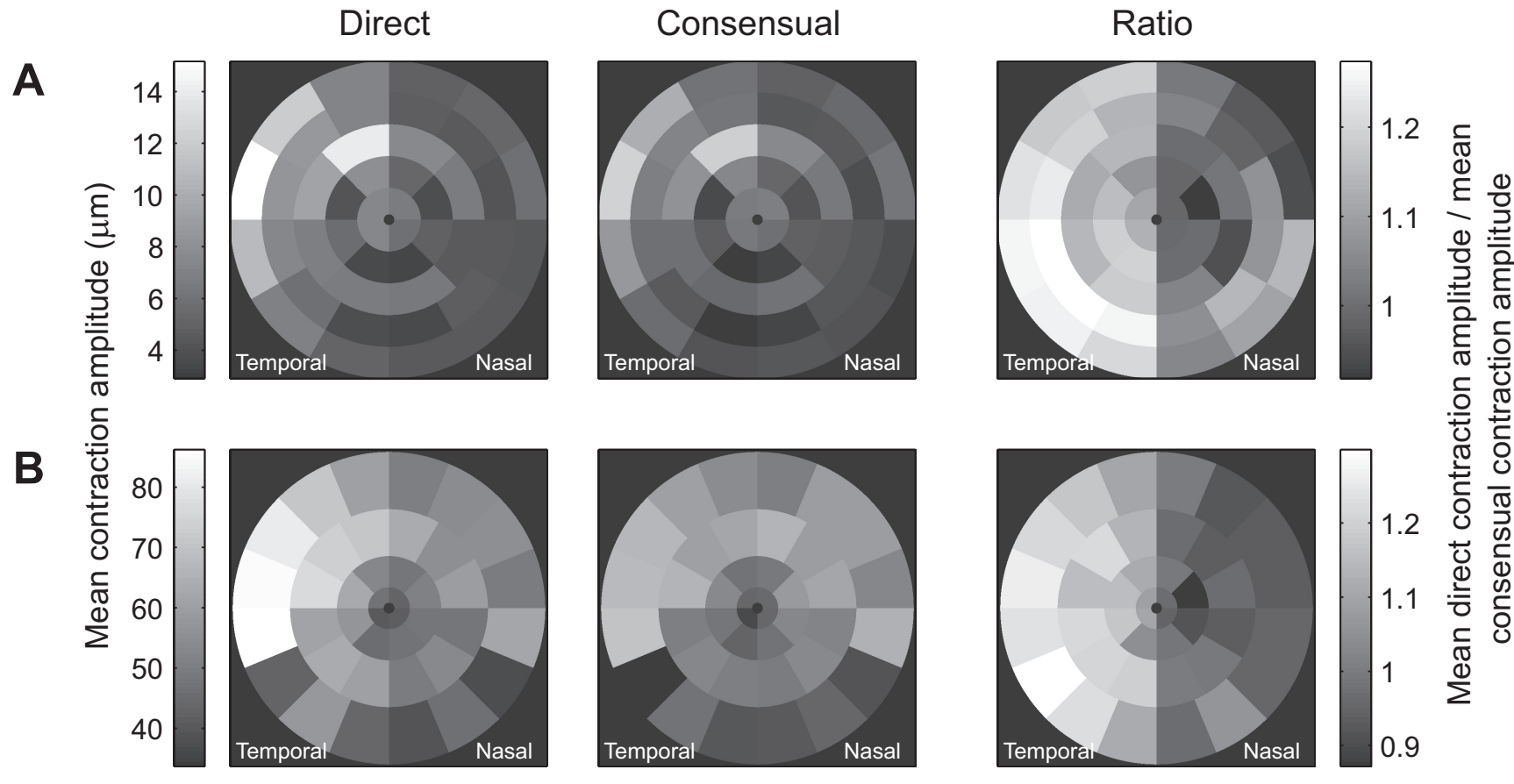

FIGURE 2. Mean pupillary contraction amplitudes and direct/consensual ratios for two representative stimulus protocols, $5 \mathrm{~A}$ (A) and $2 \mathrm{E}$ (B). Larger values are plotted in lighter shades. Mean amplitudes of subjects' direct responses (left) exhibit a gradient of sensitivity across the visual field; $n=$ 84 eyes (A), $n=32$ eyes (B). A similar pattern is seen in the means of consensual responses (center). In contrast, ratios between direct and consensual responses in corresponding test regions exhibit a different pattern: relatively homogeneous values were produced within each hemifield, these being consistently larger to stimulation in the temporal field (right). 


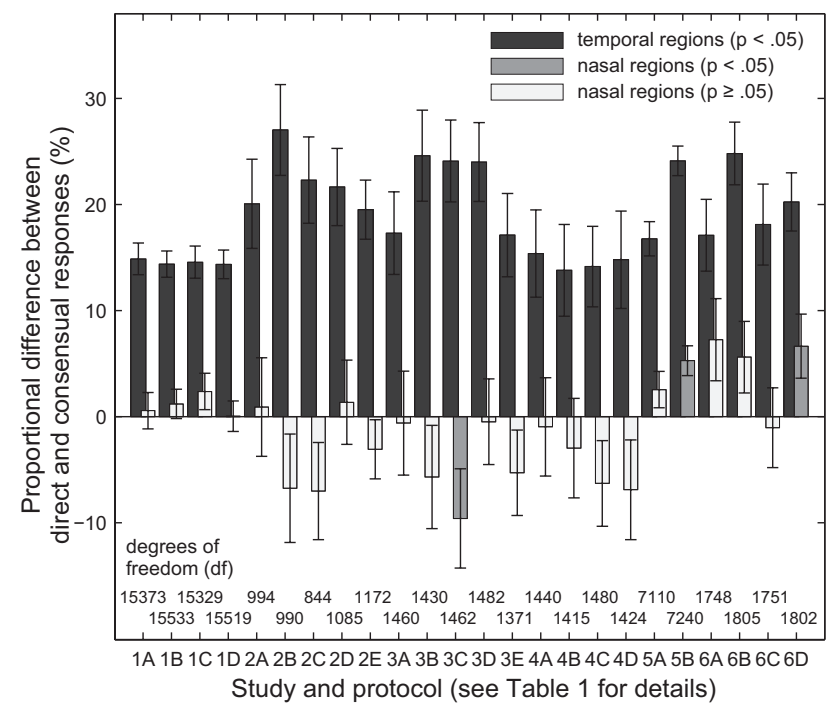

FiguRe 3. Multivariate linear analysis results showing the proportional differences between direct and consensual responses for each of the stimulus protocols. Beta values, standard errors, and degrees of freedom are plotted for the additive effect of a direct response on consensual response contraction amplitudes. These have been reported as the proportional difference between direct and consensual responses and were obtained using a multivariate linear model incorporating the effects of subject sex and age as well as regional variations in pupillary responsiveness. Positive values therefore represent larger direct than consensual responses, negative values represent larger consensual than direct. Significant differences $(P<0.05)$ were observed in the temporal field in all protocols, but only three protocols produced significant differences in the nasal field.

contraction is subject to substantial modification at various locations along the pupillary pathway. The afferent signal, carried by intrinsically photosensitive retinal ganglion cells (ipRGCs), ${ }^{13,14}$ varies in strength depending on the location of the luminance stimulus in the visual field. This pattern can be seen in the variation in contraction amplitudes between test regions in this study (Fig. 2) and largely reflects the topography of ganglion cell densities within the retina. ${ }^{13,15}$ In addition to the modulation of signal strength at various locations in the pathway, the distribution of signal at the optic chiasm and midbrain hemi-decussations creates further complexity in the resulting pattern of direct and consensual pupillary responses.

Assuming the same division of ipRGC axons at the optic chiasm as RGC axons within the main visual pathway, these cells project from homonymous hemiretinae to the corresponding pretectal olivary nucleus (PON); e.g., from right hemiretinae (left visual field of both eyes) to right PON. This means that signal from both the temporal field of the contralateral eye and the nasal field of the ipsilateral eye arrives at each PON. From the PON, intercalated neurons project to ipsilateral and contralateral Edinger-Westphal nuclei (EWN). The measurement of direct and consensual responses in many locations of the visual field therefore has the potential to provide information regarding the nature of the distribution of signals originating in different regions of the retina, between these midbrain projections.

The uniformity of the direct/consensual ratios within each hemifield in this study (Fig. 2, right) implies that for given stimulus conditions, the proportional distribution of signal to each EWN is solely dependent on whether that signal originates in nasal or temporal retina. Unlike regional variations in pupillary contraction amplitudes, this distribution appears to be independent of topographic variation in retinal sensitivity or the specific location of the stimulus within a given hemifield. The larger proportional difference between direct and consensual responses in temporal field compared with nasal (Fig. 3) is consistent with the majority of pupillary investigations of this temporo-nasal asymmetry. ${ }^{4-6}$

Anatomic studies in primates have provided evidence of asymmetry in the bilateral projections to the EWN, favoring the decussating pathway. ${ }^{16-18}$ Distribution of both temporal and nasal signals based solely on this weighting would result in equivalent but opposite ratios in each hemifield, since the more substantial projection to contralateral EWN produces the direct response when originating in nasal retina, and the consensual response when originating in temporal retina. This infers larger consensual than direct responses in the nasal field, quite different from the equivalent nasal responses we have observed. Larger consensual than direct nasal responses have been reported by others, ${ }^{3,7}$ this being however, clearly at odds with the results presented here and therefore warranting explanation.

We have previously reported significant response saturation for stimuli having higher luminance levels, at a range of presentation rates. ${ }^{19}$ Therefore, one explanation for reports of larger consensual than direct responses in the nasal field may arise from the use in those studies ${ }^{3,7}$ of high-luminance, largearea stimuli that are more likely to drive the system to maximal, saturated responses. Studies reporting results like those of this current analysis (i.e., direct/consensual $=1$ in the temporal field) tended to employ stimuli that would be less saturating, ${ }^{4-6}$ like most of the stimuli used here. This suggests that ranking the effect sizes from the linear model (Fig. 3) using an estimate of the total retinal input to the PON may provide a rationale for these apparently discordant results.

Mean contraction amplitudes for each study were used as a starting point for this estimate of input to the PON, since when holding all other variables constant, responses to these afferent inputs increase linearly with the $\log$ of stimulus luminance. ${ }^{20}$ Mean amplitudes were multiplied by a measure of stimulus field intensity, comprising an approximation of the total luminance delivered to the entire visual field over a given period (see Methods). These field intensity values ranged between 1.0 (protocol 4C) and 43.2 (protocol 1B). The resulting composite

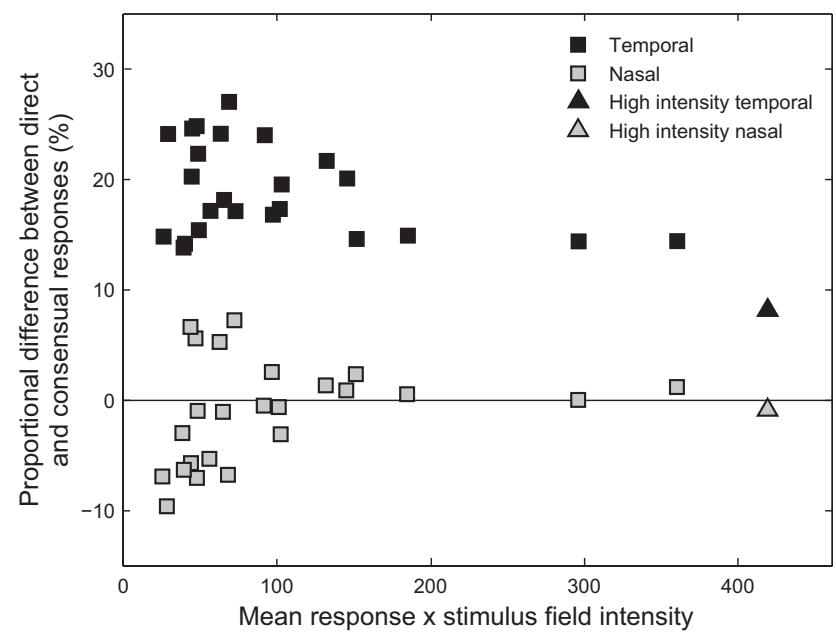

Figure 4. Proportional differences between direct and consensual responses ranked using an estimate of total retinal input to the pretectal olivary nucleus. This estimate was calculated by multiplying the mean regional standardized contraction amplitudes by an approximation of the luminance delivered to the entire visual field, referred to as the stimulus field intensity. Proportional differences from the linear model are plotted against this composite measure. Also plotted are the values for a high intensity stimulus ( $\boldsymbol{\Delta}$ see text for details). 
measure therefore provided an estimate of the afferent input to the PON during a stimulus pulse, not just from the region stimulated but also from the rest of the visual field. Figure 4 shows the proportional differences between direct and consensual responses plotted against this measure. Also plotted are the effects for a group of 12 subjects $(5$ male, 7 female, aged $34.5 \pm 11.3 \mathrm{SD}$ ) tested using a high luminance $700 \mathrm{~cd} / \mathrm{m}^{2}, 200$ ms stimulus duration, 24 region stimulus protocol (Fig. 4, triangles). It can be seen from this plot that the difference between direct and consensual responses in the temporal field decreases with increasing stimulus intensity. In the nasal field, at lower intensities, there is tendency for consensual responses to be larger than direct, this tendency decreasing with increasing intensity until these responses become fairly equal. The existence of these trends within this data begs further investigation.

Martin et al. have proposed that for each PON of an individual there is a fixed ratio of the distribution of signal between the contralateral and ipsilateral midbrain projections. ${ }^{7}$ Given the results of other studies however, this appears to be just one component of a more complex pattern. The pattern of responses observed in our present study, as well as larger consensual than direct responses at high overall luminance levels, can be explained by a model such as that presented in Figure 5. We have shown previously that the relationship between stim- ulus intensity and pupillary contraction amplitudes is well represented by a saturating Naka-Rushton function:

$$
R(I)=\frac{R_{\max } I^{z}}{I^{z}+K^{z}}
$$

where $R(I)$ is the response at a given stimulus intensity, $R_{\max }$ represents the maximum attainable response, $K$ is the stimulus intensity at which half of the maximum attainable response is reached and $\mathrm{z}$ is equivalent to the slope of the function. ${ }^{21}$ A set of Naka-Rushton curves that replicate the pattern of responses observed in this study can be produced by parsimonious adjustment of the half saturation constants, slope, and $R_{\max }$ of temporal and nasal projections (Fig. 5)

This model proposes higher maximum response levels $\left(R_{\max }\right)$ and gain $(z)$, as well as earlier saturation $(K)$ for the contralateral projection (Fig. 5B, upper). Within this projection, signal from temporal and nasal fields varies only in the level at which half saturation is reached $(K)$. Within the ipsilateral projection, signal from both hemifields behaves identically, this similarity being consistent with the pooling of both inputs within this pathway (Fig. 5B, lower). Larger direct than consensual responses are predicted in the temporal field at all stimulus intensities (Fig. 5C, upper). In the nasal field, larger
A

$$
R(I)=\frac{R_{\max } I^{z}}{I^{z}+K^{z}}
$$

B

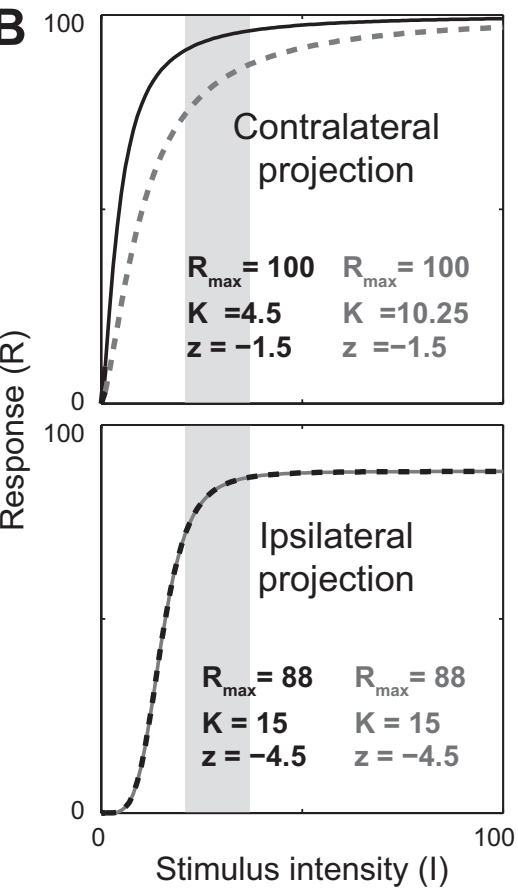

Temporal Nasal
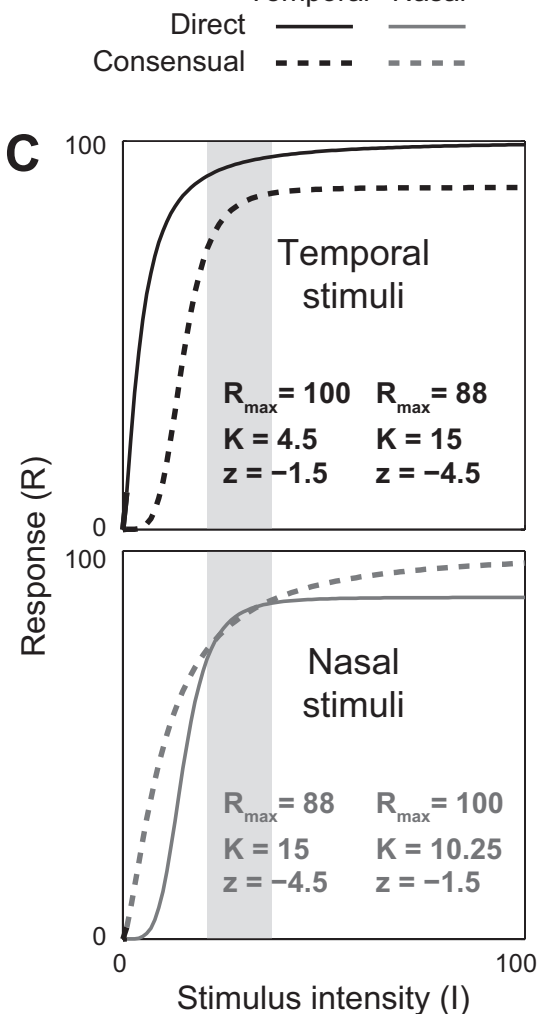

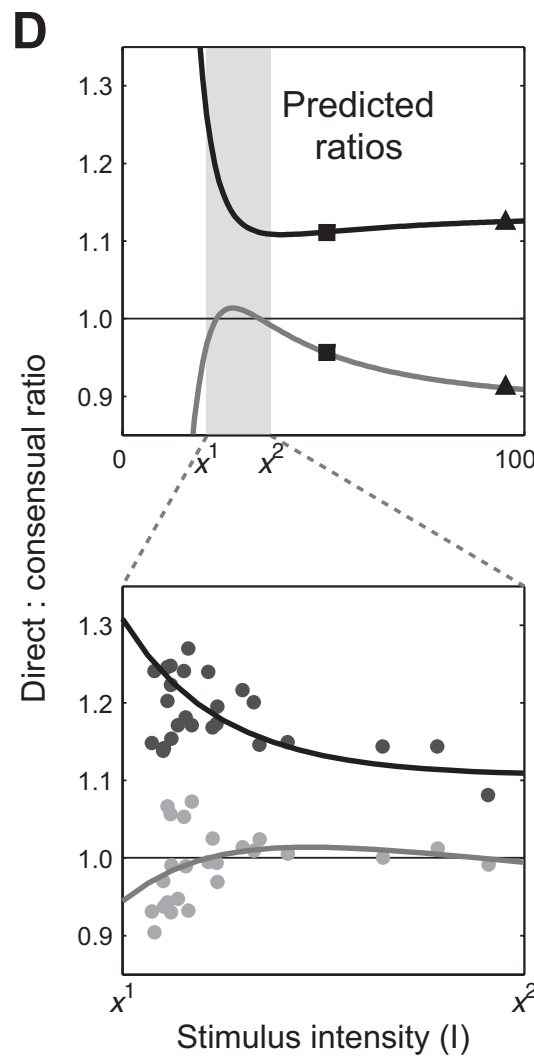

FIGURE 5. Proposed model for the distribution of retinal signal within the midbrain hemi-decussation of the pupillary pathway. Stimulus-response relationships were modeled using a series of saturating Naka-Rushton functions (A). Units for stimulus intensity (I) and response (R) in this model are arbitrary because variation between individuals is likely to result in differing values for the constants used. The shaded region in each plot represents the range of the data reported in this study. Identical functions are used for temporal and nasal field stimuli in the ipsilateral projection (B, lower). Larger $R_{\max }$, higher gain $(z)$ and earlier saturation $(K)$ are proposed for the contralateral projection (B, upper), saturation occurring at lower stimulus intensities when stimulation is in the temporal field. This pattern results in differences in the relative distribution of signal for direct and consensual pupillary contractions at different stimulus intensities and to temporal (C, upper) or nasal (C, lower) stimulation. (D) Ratios of the predicted curves for temporal and nasal stimuli shown in (C), fitted to the proportional differences between direct and consensual responses $(\mathbf{O})$, these being ranked using an estimate of retinal input to the pretectal olivary nucleus. The abscissa for this data has been scaled and shifted to allow comparison with the predicted ratios. Also shown are two pairs of hypothetical data points representing more saturated responses: direct versus consensual absolute difference nasal $<$ temporal $(\square)$ and absolute difference nasal $\approx$ temporal $(\boldsymbol{\Lambda})$. 
consensual than direct responses are predicted at high stimulus intensities (Fig. 5C, lower), the degree of this becoming more equivalent with that of temporal responses, as $R_{\max }$ is approached in this component of the contralateral projection. Predicted direct/consensual ratios produced using the proposed stimulus-response functions (Fig. 5D, upper) fit the proportional effects from the linear model well (Fig. 5D, lower).

In addition to providing an explanation for the trends within our data, these predictions are consistent with the results of the aforementioned experiments in which larger consensual than direct responses have been observed. ${ }^{3,7}$ Two plausible locations for these types of more saturated responses have been plotted on the ratio curves (Fig. 5D, upper). The first case indicates where some degree of difference is present in the nasal field, but not to the same extent as temporal, and the second a more extreme case where the differences are more equivalent. The occurrence of a relative afferent pupil defect (RAPD) due to a post-chiasmal lesion can also be explained, the differing dynamics of the ipsilateral and contralateral projections creating the large differences in sensitivity between the two eyes in the manner described by Kardon et al. ${ }^{22}$

The different levels of complexity for the two projections in this model are not incongruous with primate anatomic observations of opposing density gradients of afferent PON inputs dependent on hemiretinal origin, and the wider distribution of PON efferents projecting to the contralateral EWN than to ipsilateral. $^{18,23}$ This may suggest that the different response functions proposed for the decussating contralateral projection, are not the result of separate populations of neurons, but rather the effect of recruitment of additional neurons when the signal originates in the temporal field. This idea is further supported by the existence of PON neurons with ocular dominance restricted to, or weighted toward, the contralateral eye (i.e., the temporal field). ${ }^{24}$ Further cells recorded in that study by Clarke et al. ${ }^{24}$ responded equally well to stimulation from either eye, and it may be that the decussating EWN projection is composed of both these and the contralaterally dominated type. A subset of these cells with no ocular preference also provide a likely source for the non-decussating projection to the ipsilateral EWN.

The idea of slightly differing response dynamics in these midbrain projections depending on retinal origin, presents some challenges and opportunities for diagnostic methods using pupil responses. The use of the mean of the direct and consensual responses obtained using whole field saturating stimuli, such as in the assessment of a RAPD, may result in inaccurate estimations of dysfunction due to the differing rates of gain and saturation within these midbrain pathways. Similarly, stimuli that target the most sensitive region of the response curves will, at the same stimulus intensities, have responses that vary at different rates in the presence of reduced input. These examples suggest that improved diagnostic accuracy may be achieved simply by using measurements of both direct and consensual responses to dimmer, hemifield, or smaller stimuli. This would therefore allow adjustments to be made for the differing dynamics of the pathways contributing to these responses.

This study has demonstrated that the distribution of signal to each EWN differs depending on the hemiretina of origin, at the same time being independent of the specific location within that hemiretina. It also provides the first evidence in humans of the summation of retinal signal at the pretectal olivary nucleus in the pupillary pathway. The proposed model suggests that signal in the projection to the ipsilateral EWN, comprised of nasal field direct and temporal field consensual responses, is likely to be mediated by a single population of neurons. The projection to the contralateral EWN, responsible for the temporal field direct and nasal field consensual responses, demonstrates differing response dynamics dependent on retinal origin. This may indicate the recruitment of additional neurons for signal originating in the nasal retina. These findings have implications for the assessment of neural dysfunction using pupillary responses, since differing rates of saturation and gain impact on the accurate comparison between these different response components. Separate assessment of direct and consensual responses at hemifield or better resolution is therefore recommended in the assessment of neural dysfunction involving pupillary pathways.

\section{Acknowledgments}

The authors thank Maria Kolic, Xin-Lin Goh, and Yik-Wen Loh for their assistance in data collection and recruitment of subjects; Maria Kolic and Rohan Essex for clinical assessments; and the participants for their time and cooperation.

\section{References}

1. Smith SA, Ellis CJ, Smith SE. Inequality of the direct and consensual light reflexes in normal subjects. Br J Opbthalmol. 1979;63:523527.

2. Lowenstein O. Alternating contraction anisocoria; a pupillary syndrome of the anterior midbrain. AMA Arch Neurol Psychiatry. 1954;72:742-757.

3. Cox TA, Drewes CP. Contraction anisocoria resulting from halffield illumination. Am J Opbthalmol. 1984;97:577-582.

4. Schmid R, Wilhelm B, Wilhelm H. Naso-temporal asymmetry and contraction anisocoria in the pupillomotor system. Graefes Arch Clin Exp Opbthalmol. 2000;238:123-128.

5. Wyatt HJ, Musselman JF. Pupillary light reflex in humans: evidence for an unbalanced pathway from nasal retina, and for signal cancellation in brainstem. Vision Res. 1981;21:513-525.

6. Smith SA, Smith SE. Contraction anisocoria: nasal versus temporal illumination. Br J Ophthalmol. 1980;64:933-934.

7. Martin TL, Kardon R, Thompson HS. Unequal direct and consensual pupillary responses to hemiretinal stimuli. Invest Ophthalmol. 1991;32:1124.

8. Maddess T, Bedford S, Goh XL, James AC. Multifocal pupillographic visual field testing in glaucoma. Clin Experiment Opbthalmol. 2009;37:678-686.

9. Bell A, James AC, Kolic M, Essex RW, Maddess T. Dichoptic multifocal pupillography reveals afferent visual field defects in early type 2 diabetes. Invest Ophthalmol Vis Sci. 2010;51:602608.

10. Ruseckaite R, Maddess T, Danta G, Lueck CJ, James AC. Sparse multifocal stimuli for the detection of multiple sclerosis. Ann Neurol. 2005;57:904-913.

11. Anderson AJ, Johnson CA. Frequency-doubling technology perimetry and optical defocus. Invest Ophthalmol Vis Sci. 2003;44: 4147-4152.

12. James AC. The pattern-pulse multifocal visual evoked potential Invest Opbthalmol Vis Sci. 2003;44:879-890.

13. Dacey DM, Liao HW, Peterson BB, et al. Melanopsin-expressing ganglion cells in primate retina signal colour and irradiance and project to the LGN. Nature. 2005;433:749-754.

14. Gamlin PD, McDougal DH, Pokorny J, Smith VC, Yau KW, Dacey DM. Human and macaque pupil responses driven by melanopsin-containing retinal ganglion cells. Vision Res. 2007;47:946954.

15. Curcio CA, Allen KA. Topography of ganglion cells in human retina. J Comp Neurol. 1990;300:5-25.

16. Tigges J, O'Steen WK. Termination of retinofugal fibers in squirrel monkey: a re-investigation using autoradiographic methods. Brain Res. 1974;79:489-495.

17. Gamlin PD, Clarke RJ. The pupillary light reflex pathway of the primate. J Am Optom Assoc. 1995;66:415-418.

18. Hutchins B, Weber JT. The pretectal complex of the monkey: a reinvestigation of the morphology and retinal terminations. J Comp Neurol. 1985;232:425-442. 
19. Maddess T, Ho Y-L, Wong S, et al. Multifocal pupillographic perimetry with white and colored stimuli. J Glaucoma. In press.

20. Gamlin PD, Zhang H, Clarke RJ. Luminance neurons in the pretectal olivary nucleus mediate the pupillary light reflex in the rhesus monkey. Exp Brain Res. 1995;106:169-176.

21. Sabeti F, James AC, Maddess T. Spatial and temporal stimulus variants for multifocal pupillography of the central visual field. Vis Res. 2011;51:303-310.
22. Kardon R, Kawasaki A, Miller NR. Origin of the relative afferent pupillary defect in optic tract lesions. Ophthalmology. 2006;113:1345-1353.

23. Clarke RJ, Blanks RH, Giolli RA. Midbrain connections of the olivary pretectal nucleus in the marmoset (Callithrix jacchus): implications for the pupil light reflex pathway. Anat Embryol (Berl). 2003;207:149-155.

24. Clarke RJ, Zhang H, Gamlin PD. Primate pupillary light reflex: receptive field characteristics of pretectal luminance neurons. J Neurophysiol. 2003;89:3168-3178. 